\title{
Determining the extent of labeling for tetramethylrhodamine protein conjugates
}

\author{
David L. Meadows, Jules S. Shafer * and Jerome S. Schultz ** \\ Department of Chemical Engineering, Unicersity of Michigan, Ann Arbor, MI 48109, U.S.A.
}

(Received 25 January 1991, revised received 21 June 1991, accepted 24 June 1991)

A new, relatively simple, spectrophotometric technique has been developed which is useful for accurately determining the extent of chromophore labeling of proteins. Often the absorbance spectra and extinction coefficients of dye/protein conjugates are strongly affected by changes in the chromophore microenvironment that may occur at high dye/protein ratios. In the method being presented, the microenvironment effects have been significantly reduced by denaturing the dye /protein complex in $6 \mathrm{M}$ guanidine hydrochloride prior to making the necessary spectrophotometric measurements. With this approach, extinction coefficients were obtained under native and denatured conditions for tetramethylrhodamine isothiocyanate (TRITC) when bound to a model protein receptor, the sugar binding protein concanavalin A (ConA). The extinction coefficients used for TRITC/ConA conjugates under native and denaturing conditions were $6.52 \times 10^{4} \mathrm{M}^{-1} \mathrm{~cm}^{-1}$ and $6.96 \times 10^{4} \mathrm{M}^{-1} \mathrm{~cm}^{-1}$, respectively. These values were obtained from a model dye complex formed between TRITC and $\epsilon$-amino- $n$-caproic acid which closely resembles the sidechain of lysine residues. Additional dye/ConA conjugates were prepared with tetramethylrhodamine succinimidyl ester (RHS) and eosin isothiocyanate (EITC), and the effects of microenvironment changes on these conjugates were examined. Extinction coefficients for these dyes in native and denaturing conditions, as a function of the degree of labeling, were not appreciably different indicating that changes in the microenvironment did not have a significant affect on the spectral properties of these two dyes. In summary, with this new approach it is quite easy to accurately determine the dye/protein ratio for TRITC conjugates. Also, it is expected that RHS would be a better dye than TRITC for protein conjugation because more accurate values for dye/protein ratios can be obtained under native conditions.

Key words: Concanavalin A; Fluorescence; Labeling; Rhodamine; Eosin

\footnotetext{
Correspondence to: D. Meadows, Allergan Pharmaceuticals, Inc., 2525 Dupont Drive, Irvine, CA 92715, U.S.A.

* Present address: Department of Biochemistry, University of Michigan, Ann Arbor, MI, 48109

** Present address: University of Pittsburgh, 911 William Pitt Union, Pittsburgh, PA 15260

Abbreciations: ACA, $\varepsilon$-amino- $n$-caproic acid; ConA, concanavalin A; DMSO, dimethyl sulfoxide; EDTA, ethylenediaminetetraacetic acid; EITC, eosin isothiocyanate; $\mathrm{GuHCl}$, guanidine hydrochloride; PBS, phosphate buffered saline; ${ }_{i}$ ConA, concanavalin A protomer; RHS, tetramethylrhodamine succinimidyl ester; Succ-ConA, succinylated concanavalin A; TRITC, tetramethylrbodamine isothiocyanate.
} 


\section{Introduction}

Covalent labeling of proteins with chromophores is most frequently accomplished by reacting a chromophore containing an isothiocyanate, hydroxysuccinate ester, activated carboxylic acid, or sulfonyl chloride moiety with the $\varepsilon$-amino groups or sulfhydryl groups of a protein. Sulfhydryl groups can be labeled independent from the $\varepsilon$-amino groups by carefully using iodoacetamide or maleimide derivatives (Stryer, 1978). It is important to note that spectral properties such as extinction coefficient and quantum yield of an unconjugated dye can be significantly altered upon reaction with proteins. Chen (1969) suggested that the effects from the degree of labeling on the extinction coefficient and/or quantum yield is primarily due to changes in the microenvironment surrounding the chromophores.

Several procedures have been routinely used for determining the dye/protein ratios, but certainly the most common approach is based on spectrophotometric measurements. First, the protein concentration is calculated from the absorbance and the extinction coefficient at $280 \mathrm{~nm}$. Likewise, the dye concentration is calculated from the absorbance and extinction coefficient at the absorbance maximum of the dye (Wells et al., 1966; The and Feltkamp, 1970). This approach usually works quite well for relatively low dye to protein ratios, and for dyes with absorbance spectra that are relatively unaffected by the degree of labeling.

For chromophores such as tetramethylrhodamine isothiocyanate (TRITC), accurate determination of dye/protein ratios has proven to be quite difficult. Bergquist and Nilsson (1974) observed that the amorphous and crystalline forms of TRITC had quite different absorbance spectra when individually conjugated to antibodies. Crystalline TRITC had a single peak at $555 \mathrm{~nm}$ while amorphous TRITC had two peaks of approximately the same size at 515 and $550 \mathrm{~nm}$. The emission maximum for both amorphous and crystalline TRITC was $580 \mathrm{~nm}$, but the $515 \mathrm{~nm}$ peak did not excite the fluorescence dipole for the amorphous dye conjugate. As a result, values for the $550 \mathrm{~nm} / 515 \mathrm{~nm}$ ratio are often used as a measure of the degree of labeling (Ceba and Goldstein, 1965; Amante et al., 1972). Ravdin and Axelrod (1977) were able to separate TRITC conjugates of $\alpha$-bungarotoxin having low dye/ protein ratios (zero, one, and two TRITCs per protein molecule) using ion exchange chromatography and isoelectric focusing, but they were unable to isolate the conjugates with higher dye/protein ratios. Currently, satisfactory methods do not exist for determining high degrees of labeling for dyes such as TRITC that are very sensitive to changes in the microenvironment.

An accurate spectroscopic technique has been developed for determining the number of chromophore molecules bound to a model protein, a succinylated derivative of the jack bean lectin concanavalin A. The succinylated derivative was used because succinylation causes the tetrameric native ConA to separate into two stable, non-aggregating dimers (Gunther et al., 1973). Results are also presented from the labeling of ConA with rhodamine derivatives that contain a succinimidyl ester moiety instead of an isothiocyanate moiety. Finally, results are given that show how the labeling extent affects the spectra of eosin isothiocyanate which is known to be relatively insensitive to microenvironment changes when bound to proteins.

\section{Materials and methods}

Spectroscopic grade guanidine hydrochloride $(\mathrm{GuHCl})$, concanavalin A (ConA), $\varepsilon$-amino- $n$ caproic acid (ACA), dimethyl sulfoxide (DMSO), and tetramethylrhodamine-5[and-6] isothiocyanate labeled ConA (TRITC/ConA) were obtained from Sigma Chemical (St. Louis, MO). ${ }^{14} \mathrm{C}$-succinic anhydride was from ICN Radiochemicals (Irvine, CA). Tetramethylrhodamine isothiocyanate, 5,6-carboxytetramethylrhodamine succinimidyl ester (RHS), and eosin-5-isothiocyanate (EITC) were from Molecular Probes (Eugene, OR). All of the reagents and materials were used without further purification. Dialysis bags were from Spectrum Medical Industries (Los Angeles, CA.). The chemical structure of the chromophores used in this study are shown in Fig. 1. 
<smiles>CN(C(=O)[O-])c1ccc(C(=O)[O-])cc1</smiles><smiles></smiles>

B<smiles></smiles>

C

Fig. 1. The chemical structures of chromophores used for labeling. $A$ : tetramethylrhodamine-5 (and -6 ) isothiocyanate (TRITC); $B$ : eosin-5-isothiocyanate (EITC). $C$ : tetramethylrhodamine succinimidyl ester (RHS).

An SLM (Urbana, IL) SPF-500C spectrofluorometer was used for all fluorescence measurements, and a Varian Instruments (Palo Alto, CA) DMS $200 \mathrm{UV}$-Vis spectrophotometer was used to collect all absorbance spectra.

\section{Basis of methods}

The principal difficulty in determining the extent of labeling for TRITC conjugates is due to the effects of changes in the protein microenvironment on the absorbance spectra of the bound molecules of TRITC. The procedure being presented is capable of accurately determining TRITC/protein ratios and is similar to the approach used by Edelhoch (1967) to estimate the number of tryptophan and tyrosine residues in proteins. Edelhoch developed a procedure based on absorbance spectrometry in which he was able to denature proteins in a $6 \mathrm{M} \mathrm{GuHCl}$ solution thereby significantly reducing the effects of microenvironment changes on the absorbance properties of tryptophan and tyrosine residues. By destroying the secondary and tertiary structure of the protein (Haschemeyer and Haschemeyer, 1973), the number of tryptophan and tyrosine residues in the denatured protein was accurately determined using standard spectroscopic techniques. For proteins containing more than one sulfhydryl group, a thio reagent such as $\beta$ mercaptoethanol might be required to ensure that total denaturation is achieved.

In the current approach, the Edelhoch procedure has been slightly modified so it can be applied to the determination of the dye/protein ratio for rhodamine and eosin protein conjugates. The extinction coefficient for the dye/protein conjugate was approximated by forming a model complex between the dye and ACA, which was chosen for the model complex because it closely resembles the reactive side chain of lysine residues. Under appropriate $\mathrm{pH}$ conditions, dyes containing isothiocyanate or succinimidyl ester functional groups readily react with the primary amine functional group on the lysine side chain in proteins as well as the primary amine in ACA. The extinction coefficient for the TRITC/ACA complex was assumed to be a good approximation for the extinction coefficient of the denatured TRITC/ConA conjugates. Extinction coefficients for the model compound were determined in native and denaturing conditions from which the number of TRITC moieties attached to each 
ConA protomer (pConA) was then directly calculated. Similar procedures were followed for the RHS and EITC conjugates.

\section{Dye conjugation with Succ-ConA}

Succinylated conjugates of ConA (Succ-ConA) were prepared prior to being labeled with a dye because the succinylation procedure induced several important changes in the native tetrameric ConA. By reacting the lysine residues with succinic anhydride, the positive charged lysine side chains were converted to negative charges. These changes in the overall charge distribution of the protein, combined with changes in the steric interactions, caused the native ConA tetramer molecule to separate into two identical dimers that did not re-aggregate into the tetramers even at elevated temperatures (Gunther et al., 1973). Since a dynamic equilibrium normally exists between the dimer and tetramer forms of conA, it was essential that the dimers of conA be prevented from aggregating in order to isolate the relationship between changes in the spectral properties of dyes caused by microenvironment changes and the extent of dye labeling.

$120 \mathrm{mg}$ of ConA was conjugated with ${ }^{14} \mathrm{C}$-succinic anhydride in a $30 \mathrm{ml}$ aqueous solution containing $0.15 \mathrm{M} \mathrm{NaCl}$ using a procedure described elsewhere (Meadows and Schultz, in press). $0.3 \mathrm{M}$ methyl $\alpha$-mannose was added to the solution in order to protect the carbohydrate binding sites. Using this procedure, succinylated ConA conjugates (Succ-ConA) were prepared at five different levels of succinylation. Next, a solution of 20 $\mathrm{mg}$ TRITC in $2 \mathrm{ml}$ of dry DMSO was prepared immediately prior to the dye conjugation step. 0.4 $\mathrm{ml}$ of the TRITC/DMSO solution was added to each of the five Succ-ConA samples over a period of $15 \mathrm{~min}$ at room temperature. The $\mathrm{pH}$ was maintained at 9.5 using $0.1 \mathrm{M} \mathrm{NaOH}$ with continuous stirring until the conjugation reaction was completed after $1 \mathrm{~h}$. TRITC-labeled Succ-ConA was purified using a Sephadex G-75 affinity column and eluted using $0.5 \mathrm{M}$ glucose (Agrawal and Goldstein, 1965). The most concentrated fractions collected from the affinity column were then dialyzed twice against four liters of $0.05 \mathrm{M}$ phosphate buffer saline (PBS) at $\mathrm{pH} 7.4$ to remove the glucose.
Conjugates of RHS and EITC were synthesized using the same procedure outlined for TRITC conjugation except that $25 \mathrm{mg}$ of RHS was dissolved in $2.0 \mathrm{ml}$ of dry DMSO, and $33 \mathrm{mg}$ of EITC was dissolved in $2.0 \mathrm{ml}$ of dry dimethylformamide prior to being added to the five SuccConA solutions.

\section{Reaction of TRITC with $\epsilon$-amino-n-caproic acid}

$3.0 \mathrm{mg}$ of ACA was added to $2.0 \mathrm{ml}$ of deionized water and the $\mathrm{pH}$ was adjusted to 9.5 using $0.1 \mathrm{M} \mathrm{NaOH} .1 .23 \mathrm{mg}$ of TRITC was dissolved in $0.1 \mathrm{ml}$ dry DMSO and the solution was immediately added to the ACA solution at room temperature with agitation. The final mole ratio of TRITC to ACA was 1:8 with a final TRITC concentration of $1.35 \mu \mathrm{M} / \mathrm{ml}$. The $\mathrm{pH}$ was maintained at 9.5 with $0.1 \mathrm{M} \mathrm{NaOH}$, and the reaction was complete after $1 \mathrm{~h}$.

\section{Purity of TRITC raw material}

A reverse phase HPLC assay method was developed to determine the purity of the TRITC raw material based on the amount of TRITC which reacts with ACA under the conjugation reaction conditions. The method was also used to confirm that the competing hydrolysis reaction rates for the dyes are much slower than the protein conjugation reaction rates. Three samples were prepared and assayed at three time points: 0,6 , and $24 \mathrm{~h}$. The first sample was the TRITC raw material dissolved in anhydrous DMSO followed by dilution into $10 \mathrm{ml}$ of dry methanol. Sample number two was the product from the TRITC/ACA conjugation reaction. The final sample contained $50 \mu \mathrm{l}$ of the TRITC/DMSO stock solution which had been diluted to $10 \mathrm{ml}$ using $0.02 \mathrm{M}$ phosphate buffer at $\mathrm{pH} 7.0$. The $\mathrm{pH}$ 7.0 reaction conditions were selected as a worst case scenario for the competing hydrolysis reaction given that hydrolysis of TRITC is significantly more rapid at $\mathrm{pH} 7.0$ than at 9.5 while the reaction between ACA and TRITC is much more rapid at $\mathrm{pH} 9.5$ than at 7.0.

The HPLC assay method used to resolve the important reaction products had a mobile phase of $70 \%$ methanol and $30 \% 0.02 \mathrm{M}$ phosphate buffer at a flow rate of $1.5 \mathrm{ml} / \mathrm{min}$ with an injection volume of $20 \mu \mathrm{l}$ and a wavelength of 280 
$\mathrm{nm}$. The column used was a Waters $\mu$ Bondapac $^{\mathrm{TM}} \cdot \mathrm{c} 18(25 \mathrm{~cm})$. The HPLC system consisted of a Beckman 506 auto-sampler, a 167 scanning detector, and a 116 solvent module. Under these operating conditions, the retention time for TRITC impurities were 3.8 and $11.5 \mathrm{~min}$ while the retention times for the active raw material were 5.6 and $7.4 \mathrm{~min}$. Absorbance spectra were taken of each elution peak to confirm the characteristic tetramethylrhodamine absorbance spectra which indicated that the peak was indeed either a reactive or nonreactive form of TRITC.

\section{Spectral properties of dye / protein conjugates}

The absorbance spectra of all dye/protein conjugates were taken under native (0.05 M PBS, $\mathrm{pH}$ 7.4) and denaturing conditions (6 M GuHCl). Denatured dye/protein conjugate samples were prepared as follows. A solution of $7.1 \mathrm{M} \mathrm{GuHCl}$ was adjusted to $\mathrm{pH} 7.4$ using $0.1 \mathrm{M} \mathrm{NaOH} .3 .0 \mathrm{ml}$ of the $\mathrm{GuHCl}$ solution was added to $0.5 \mathrm{ml}$ of the dye /ACA or dye/protein solution with vigorous agitation to give the final $6 \mathrm{M} \mathrm{GuHCl}$ concentration. Absorbance measurements were then made on the conjugates, and extinction coefficients calculated from these measurements. Protein concentrations were determined by a modified Folin phenol method (Ohnishi and Barr, 1978) which had been independently calibrated to total protein concentrations based on amino acid analyses.

\section{Preparation of dialysis membranes}

Dialysis bags with a molecular weight cut off of $12-14 \mathrm{kDa}$ and $8 \mathrm{kDa}$ were prepared for use by first heating in a $2 \%$ sodium carbonate solution to $60^{\circ} \mathrm{C}$ followed by washing in deionized water. The bags were then heated in deionized water to $60^{\circ} \mathrm{C}$ followed by heating in $1 \mathrm{mM}$ EDTA. The treated bags were stored in $1 \mathrm{mM}$ EDTA at $4^{\circ} \mathrm{C}$ (Edelman and Wang, 1978).

\section{Results}

\section{Purity of TRITC raw material}

A sample containing the TRITC raw material dissolved in DMSO and diluted with methanol had two peaks at 3.8 and $11.5 \mathrm{~min}$ for the inactive fractions which constituted approximately $8 \%$ of the total chromatogram area. This was true after $24 \mathrm{~h}$ also, indicating that the TRITC raw material was quite stable when stored in a dry organic solvent. The $8 \%$ impurity was also evident in the second sample which contained the TRITC/ACA conjugation product. For this sample, the TRITC/ACA product eluted with the solvent front while the size and retention times of the impurity were unchanged. The HPLC chromatogram for the third sample containing TRITC raw material in $\mathrm{pH}$ 7.0 PBS indicated that the impurity peaks were probably due to hydrolysis products of the raw material. After $24 \mathrm{~h}$ in the aqueous solution, approximately $16 \%$ of the rhodamine was contained in the impurity peaks that eluted at 3.8 and $11.5 \mathrm{~min}$. In summary, these results clearly indicate that the TRITC raw material contains approximately $8 \%$ of an impurity that can not react with ACA or with protein during the conjugation procedure. Also, the competing hydrolysis reaction is sufficiently slow that it is negligible during the time required for the conjugation reaction to reach completion.

\section{Extinction coefficients for TRITC / ACA product}

The absorbance spectra of the TRITC/ACA conjugate in PBS and $6 \mathrm{M} \mathrm{GuHCl}$ are shown in Fig. 2 along with the excitation spectrum for the TRITC raw material in PBS. The absorbance maximum for both spectra in PBS was at $551 \mathrm{~nm}$ while the maximum in $\mathrm{GuHCl}$ was shifted to longer wavelengths by $5 \mathrm{~nm}$. There were only slight differences in the general shapes of all three spectra. From the known concentration of the TRITC/ACA stock solution $\left(13.5 \times 10^{-6} \mathrm{M}\right)$ and the peak absorbances in PBS and $\mathrm{GuHCl}$ ( 0.81 and 0.864 , respectively), the extinction coefficients were calculated to be $6.52 \times 10^{4}$ and 6.96 $\times 10^{4} \mathrm{M}^{-1} \mathrm{~cm}^{-1}$, respectively. These extinction coefficients are corrected for the $8 \%$ impurity present in the TRITC raw material.

\section{Determining dye / protein ratios}

Dye/protein ratios have normally been determined directly from the absorbance of the protein and the conjugated dye under native conditions, but this approach can result in quite large errors. For example, with the extinction coefficient supplied by the manufacturer and the ab- 


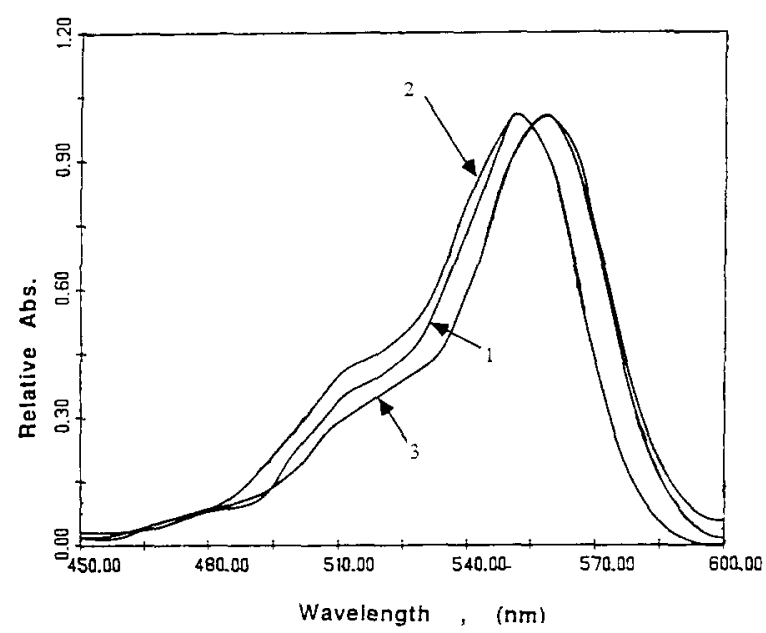

Fig. 2. The absorbance and fluorescence excitation spectra for TRITC and the TRITC/ACA model compound. (1) Excitation spectrum of TRITC raw material in PBS $\left(\lambda_{\mathrm{cx} \max }=551\right.$ $\left.\mathrm{nm}, \lambda_{\mathrm{cml}}=620 \mathrm{~nm}\right) ;(2)$ absorbance spectrum for TRITC/ACA

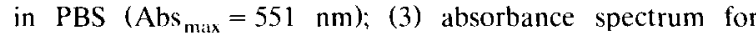
TRITC/ACA in $6 \mathrm{M} \mathrm{GuHCl}\left(\mathrm{Abs}_{\mathrm{mix}}=556 \mathrm{~nm}\right)$.

sorbance at $551 \mathrm{~nm}$ for the TRITC/ConA conjugates, it is possible to estimate the extent of labeling for the dye/protein conjugates under native conditions. The spectra of TRITC/ConA conjugates in PBS are shown in Fig. 3, curves $1-4$. They are quite similar to spectra presented by Ravdin and Axelrod (1977) in that there are two major absorbance peaks that significantly overlap, one at $518 \mathrm{~nm}$ and another at $551 \mathrm{~nm}$. The relative heights of the two peaks were strongly dependent on the extent of TRITC labeling, therefore, determination of dye/protein ra-

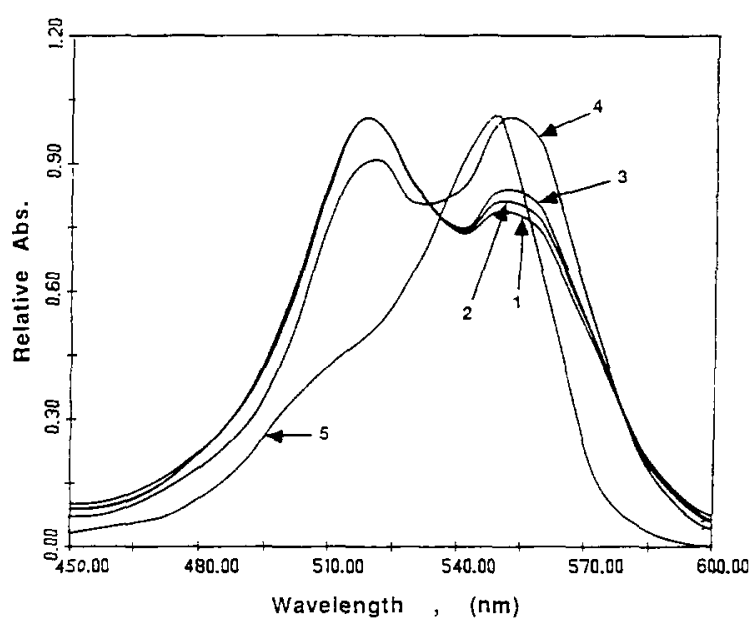

Fig. 3. The absorbance spectra for TRITC/ConA conjugates under native conditions in PBS, see Table I. (1) Sample 0, $551 / 518$ ratio $=0.78 ;(2)$ sample $2,551 / 518$ ratio $=0.81 ;$ (3) sample $3,551 / 518$ ratio $=0.85 ;$ (4) sample $4,551 / 518$ ratio $=$ 1.11; (5) TRITC raw material in PBS.

tios directly from the absorbance spectra obtained under native conditions would have caused significant errors. Also, it is obvious from the excitation spectrum in Fig. 2 that the $518 \mathrm{~nm}$ peak does not excite the fluorescence dipole so the $551 \mathrm{~nm}$ peak is the peak that is most relevant to dye conjugation measurements.

In the second and third columns of Table I, results are given for five TRITC/ConA conjugates under native conditions. The ratio of the peak absorbances at 551 and $518 \mathrm{~nm}$ were calculated as well as the dye/protein ratios, which were determined using conventional methods with

\section{TABLE I}

\section{RESULTS FOR CONA CONJUGATION WITH SUCCINIC ANHYDRIDE AND TRITC}

The calculations of the TRITC/pConA ratios for native and denatured conjugates are based on extinction coefficients of $6.52 \times 10^{4}$ and $6.96 \times 10^{4} \mathrm{M}^{-1} \mathrm{~cm}^{-1}$, respectively. The average number of lysine residues occupied on each ConA protomer by either a succinate or TRITC moiety is given in the 'Total' column. There are 12 available lysine residues in each protomer.

\begin{tabular}{|c|c|c|c|c|c|c|}
\hline \multirow[t]{2}{*}{ Sample } & \multicolumn{2}{|c|}{ Native conditions (PBS) } & \multicolumn{2}{|c|}{ Denaturing (GuHCl) } & \multirow{2}{*}{$\begin{array}{l}\text { Ratio of } \\
\text { Succ/pConA }\end{array}$} & \multirow[t]{2}{*}{ Total } \\
\hline & $551 / 518$ & $\overline{\mathrm{TRITC} / \mathrm{pCon} \mathrm{A}}$ & $556 / 525$ & TRITC/pConA & & \\
\hline$\overline{0}$ & 0.78 & 4.3 & 1.40 & 6.4 & 0.0 & 6.4 \\
\hline 1 & 0.82 & 3.6 & 1.38 & 7.6 & 1.2 & 8.8 \\
\hline 2 & 0.81 & 1.2 & 1.30 & 6.2 & 2.8 & 9.0 \\
\hline 3 & 0.85 & 1.9 & 1.35 & 4.7 & 4.7 & 9.4 \\
\hline 4 & 1.11 & 3.4 & 1.74 & 2.8 & 7.3 & 10.1 \\
\hline
\end{tabular}




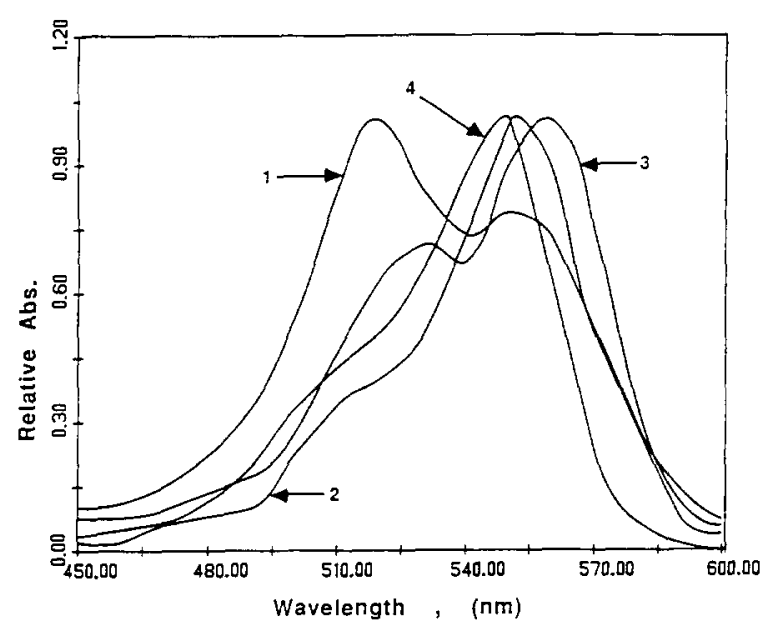

Fig. 4. Comparison of the absorbance spectra for TRITC/ ConA conjugates in PBS and in $6 \mathrm{M} \mathrm{GuHCl}$, see Table I. (1) Sample 0 in PBS, $551 / 518$ ratio $=0.78$; (2) excitation spectrum of TRITC in PBS $\left(\lambda_{\mathrm{ex} \max }=551 \mathrm{~nm}, \lambda_{\mathrm{cm}}=620 \mathrm{~nm}\right)$; (3) sample 0 in $6 \mathrm{M} \mathrm{GuHCl}, 556 / 525$ ratio $=1.4$; (4) TRITC raw material in PBS.

an extinction coefficient in PBS of $6.52 \times 10^{4}$ $\mathrm{M}^{-1} \mathrm{~cm}^{-1}$. These results clearly show that accurate estimates of the degree of TRITC labeling can not be made under native conditions with conventional methods because it is unclear which peak (the $551 \mathrm{~nm}$ or the $518 \mathrm{~nm}$ peak) is the best indicator for the actual degree of labeling. For example, a relatively low dye/protein ratio of 3.4 for sample 4 gave a $551 / 518$ ratio of 1.11 . On the other hand, a slightly higher dye/protein ratio of 4.3 for sample 0 gave a $551 / 518$ ratio of 0.78 . Based on the 551/518 ratio of 0.81 for sample 2, it would have been expected that the dye /protein ratio would be between 3.4 and 4.3 , but the dye/protein ratio for sample 2 under native con- ditions was calculated to be only 1.2 . From this example, as well as from previous work reported in the literature, it is clear that the conventional spectroscopic techniques normally used to determine dye/protein ratios are unacceptable for TRITC/protein conjugates.

The spectra of the TRITC/ConA conjugates were much different when the protein was denatured in $6 \mathrm{M} \mathrm{GuHCl}$ (Fig. 4). First of all, the absorbance peak for sample 0 was shifted by 5 $\mathrm{nm}$ to $556 \mathrm{~nm}$. Also, the $556 \mathrm{~nm}$ peak became significantly larger in comparison to the 518 peak. These results clearly show that the extinction coefficient of the $551 \mathrm{~nm}$ peak under native conditions was being significantly affected by changes in the microenvironment surrounding the bound dye that occurred as more dye molecules were bound to the protein. In columns 4 and 5 of Table I, the dye/protein ratios have been calculated under denaturing conditions based on the absorbance at $556 \mathrm{~nm}$ and the extinction coefficient, $6.96 \times 10^{4} \mathrm{M}^{-1} \mathrm{~cm}^{-1}$. It is clear from a comparison of column 3 and column 5 that the results obtained under denaturing conditions are significantly different from the results obtained under native conditions. For example, under native conditions the dye/protein ratio was 1.2 for sample 2, while under denaturing conditions the ratio was a factor of five higher at 6.2 .

The results summarized in Table II for RHS conjugates show that there were some significant differences between the spectral properties for TRITC and RHS. Comparison of results for samples 0 through 4 shows that even though the $556 / 520$ ratio for native conditions did not vary significantly with labeling, the RHS/pConA ratio did increase by more than a factor of three be-

TABLE II

RESULTS FOR CONA CONJUGATION WITH SUCCINIC ANHYDRIDE AND RHS

\begin{tabular}{|c|c|c|c|c|c|c|}
\hline \multirow[t]{2}{*}{ Sample } & \multicolumn{2}{|c|}{ Native conditions (PBS) } & \multicolumn{2}{|c|}{ Denaturing $(\mathrm{GuHCl})$} & \multirow{2}{*}{$\begin{array}{l}\text { Ratio of } \\
\text { Succ/pConA }\end{array}$} & \multirow[t]{2}{*}{ Total } \\
\hline & $\overline{556 / 520}$ & RHS $/ \mathrm{pConA}$ & $\overline{560 / 527}$ & $\overline{\mathrm{RHS} / \mathrm{pConA}}$ & & \\
\hline 0 & 1.20 & 5.7 & 1.56 & 8.6 & 0.0 & 8.6 \\
\hline 1 & 1.19 & 3.2 & 1.54 & 8.3 & 1.2 & 9.5 \\
\hline 2 & 1.22 & 1.6 & 1.70 & 2.3 & 2.3 & 4.6 \\
\hline 3 & 1.24 & 2.0 & 1.77 & 2.0 & 3.8 & 5.8 \\
\hline 4 & 1.32 & 1.6 & 2.02 & 1.7 & 6.6 & 8.3 \\
\hline
\end{tabular}




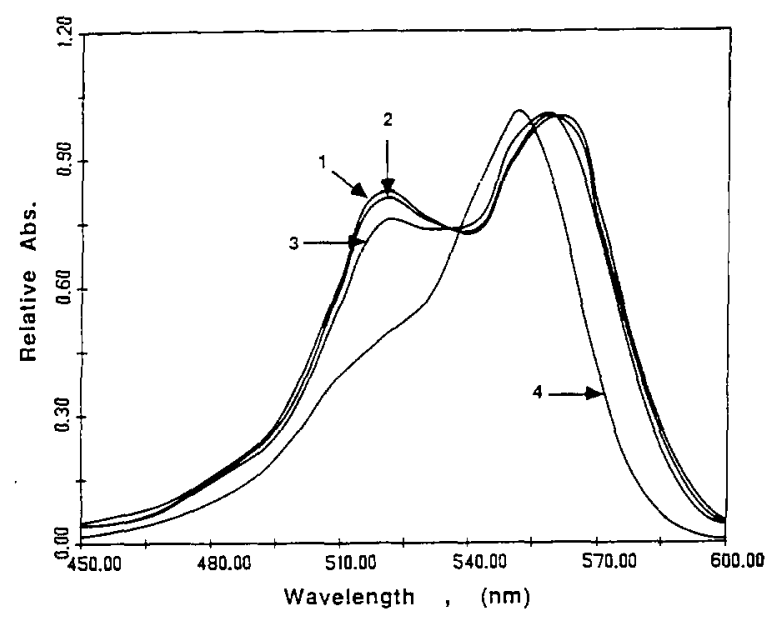

Fig. 5. The absorbance spectra for RHS/ConA conjugates under native conditions in PBS, see Table II. (1) Sample 0, $556 / 520$ ratio $=1.20 ;(2)$ sample $2,556 / 520$ ratio $=1.22 ;(3)$ sample $4,556 / 520$ ratio $=1.32$; (4) RHS raw material in PBS.

tween sample 4 and sample 0 . From these results, it would be expected that dye/protein ratios for RHS under native conditions would be closer to the ratios obtained under denaturing conditions than was observed for the TRITC conjugates. This was indeed the case as evidenced by a comparison with the values for RHS/pConA ratios in columns 3 and 5 of Table II. The absorbance spectra in Fig. 5 for RHS conjugates confirm that the extent of labeling caused only slight changes

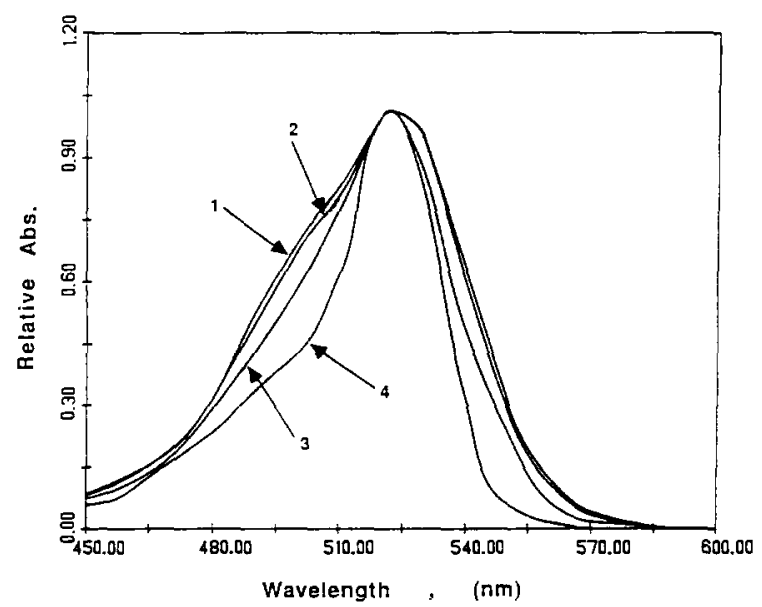

Fig. 6. The absorbance spectra for EITC/ConA conjugates under native conditions in PBS, see Table III. (1) Sample 0 $\left(\lambda_{\text {Abs max }}=522 \mathrm{~nm}\right)$; (2) sample 2; (3) sample 4; (4) EITC raw material in PBS. in the 556/520 ratio and in the overall spectral fingerprints of the dye. A general characteristic for all of the RHS/ConA samples was that the $556 \mathrm{~nm}$ peak was significantly larger relative to the $520 \mathrm{~nm}$ peak, therefore the overlap of the smaller $520 \mathrm{~nm}$ would have less total effect on the $556 \mathrm{~nm}$ peak for the RHS conjugates than was observed for TRITC. This would tend to indicate that the extinction measurements made at 556 $\mathrm{nm}$ for RHS conjugates should be more accurate than the extinction measurements at $551 \mathrm{~nm}$ for TRITC conjugates under the same native conditions.

Results given in Table II for the RHS conjugates under denaturing conditions show that the maximum absorbance peak was shifted slightly to $560 \mathrm{~nm}$, but the overall spectral shape did not change significantly. Also, the $520 \mathrm{~nm}$ peak decreased approximately $20 \%$ relative to the 560 $\mathrm{nm}$ peak as evidenced by the $560 / 527$ ratio in column 4. The decrease in the $520 \mathrm{~nm}$ peak relative to the $560 \mathrm{~nm}$ peak would be expected to further increase the accuracy of the dye/protein ratios obtained under denaturing conditions. Comparison of the RHS/pConA ratios in columns 3 and 5 of Table II shows that there was a significant difference between native and denaturing conditions for samples with the largest degree of labeling (samples 0 and 1), while samples 2,3 , and 4 were approximately the same under both conditions.

Protein conjugates of EITC were prepared in a similar fashion because eosin has been reported to be rather insensitive to the degree of dye labeling (Cherry et al., 1976). As anticipated, all of the EITC conjugates in Table III had very similar absorbance spectra under native condi-

\section{TABLE III}

RESULTS FOR CONA CONJUGATION WITH SUCCINIC ANHYDRIDE AND EITC

\begin{tabular}{lllll}
\hline Sample & Native & Denaturing & Ratio of & Total \\
& EITC/pConA & EITC/pConA & Succ/pConA & \\
\hline 0 & 8.0 & 5.6 & 0.0 & 5.6 \\
1 & 6.3 & 7.5 & 0.6 & 8.1 \\
2 & 4.7 & 4.6 & 1.6 & 6.2 \\
3 & 2.6 & 2.3 & 3.9 & 6.2 \\
4 & 1.5 & 1.7 & 6.7 & 8.4 \\
\hline
\end{tabular}


tions (Fig. 6), regardless of the degree of labeling. There was very little change in the shape of the spectra as the EITC/pConA ratio increased, and the absorbance maximum was centered at $522 \mathrm{~nm}$ for all samples. Under denaturing conditions, the absorbance maximum shifted to $527 \mathrm{~nm}$, but there were only slight changes in the overall shape of the spectra. This clearly indicated that changes in the protein microenvironment did not significantly alter the spectra, therefore, the dye/protein ratios determined under native conditions were in close agreement with dye/protein ratios determined under denaturing conditions. Columns 2 and 3 in Table III show that sample 0 was the only sample for which the EITC/pConA ratios obtained under native and denaturing conditions were not in very close agreement. From these results, accurate dye/protein ratios should be expected for protein conjugates of EITC when the dye/protein ratios are determined under native conditions using an extinction coefficient of $8.6 \times 10^{4} \mathrm{M}^{-1} \mathrm{~cm}^{-1}$ (Cherry et al., 1976).

The Succ/pConA ratios given in Tables I, II, and III provide indirect, yet supportive, evidence that the dye/protein ratios obtained in $\mathrm{GuHCl}$ were closer to the actual dye/protein values than were the values obtained under native conditions. For example, sample 1 in all three tables had virtually the same Succ/pConA ratio which would tend to indicate that all three samples had approximately the same number of free lysine sites available for dye labeling subsequent to succinylation. However, the dye/protein ratio under denaturing conditions for sample 1 in Table III (7.5) was almost twice the ratio for sample 1 in Table I under native conditions (3.6). The two ratios would be expected to be approximately the same since TRITC and EITC would be expected to have almost the same reactivity for free lysine residues in the protein. Also, a comparison between columns 2 and 3 in Table III shows that the dye/protein ratios determined under native and denaturing conditions for EITC conjugates were approximately the same value. Therefore, based on the indirect evidence supplied by the ${ }^{14} \mathrm{C}$-succinate derivatives, the results for TRITC/ protein ratios obtained under native conditions were probably too low in comparison to the EITC/protein ratios. In conclusion, conjugation of lysine residues with ${ }^{14} \mathrm{C}$-succinic anhydride provided additional evidence that dye/protein ratios obtained under denaturing conditions were significantly more accurate than ratios obtained under native conditions for both the TRITC and RHS dyes.

The synthesis of ${ }^{14} \mathrm{C}$-succinate derivatives also helped to confirm that the only available sites on the protein molecules were the 12 lysine residues in each ConA protomer. Labeling experiments in an excess of ${ }^{14} \mathrm{C}$-succinic anhydride gave a maximum of approximately 11 succinate moieties per ConA protomer. The results tended to indicate that under these labeling reaction conditions the dye molecules reacted almost exclusively to the lysine residues. It also appeared that the reaction of dye molecules with the $\alpha$-amino terminus of each protomer did not significantly affect the labeling results.

\section{Discussion}

Precise information about the extent of chromophore labeling of proteins is often not required for applications such as fluorescence microscopy, cell staining, and cell sorting. On the other hand, accurate values for dye/protein ratios are essential in areas such as quantitative immunofluorescence where fluorescence is used as an indirect measure of ligand concentration and non-radiative energy transfer where the efficiency of energy transfer is strongly affected by the donor/acceptor stoichiometry. For TRITC, the standard ratiometric absorbance procedure can not be used to determine accurate values for the dye/protein ratio. An explanation supplied in the literature for this behavior is that changes in the microenvironment around the protein-bound TRITC molecules have significant effects on the TRITC absorbance spectrum. In the current work, it has been shown that by denaturing the TRITC/ConA conjugates in $6 \mathrm{M} \mathrm{GuHCl}$, most of the microenvironmental effects on the spectral properties of TRITC are eliminated, thus making it possible to obtain accurate measurements even for high dye/protein ratios. Even though the protein is expected to assume a random coil configuration in $6 \mathrm{M} \mathrm{GuHCl}$, it is unlikely that 
the TRITC bound to the denatured protein has the same degree of solvation and the same microenvironment that surrounds TRITC when it is bound to ACA. This may help to explain why the $518 \mathrm{~nm}$ peak was not completely eliminated in denatured TRITC/ConA conjugates with high degrees of labeling. For low degrees of labeling (less than 2 dye molecules per ConA protomer) the $515 \mathrm{~nm}$ peak had very little influence on the $551 \mathrm{~nm}$ peak. This is also true for commercially prepared TRITC/ConA conjugates which typically have dye/protein ratios less than 1 .

Labeling experiments with RHS conclusively showed that the reactivity between the dye and protein, hence the extent of dye labeling, was very similar to the reactivity observed between TRITC and the protein. However, the use of RHS conjugates did have the advantages that the $520 \mathrm{~nm}$ peak was much smaller relative to the 556 nm peak, and the absorbance spectra were less sensitive to microenvironment changes than were TRITC conjugates. Therefore, it would appear that RHS would be a better dye to use than TRITC for fluorescence labeling applications where accurate dye/protein ratios must be determined under native (nondenaturing) conditions. Experiments with EITC conjugates clearly showed that EITC was relatively insensitive to environmental changes, therefore, the dye/protein ratios determined under native conditions were quite close to the ratios obtained under denaturing conditions.

\section{Acknowledgements}

This work has been partially supported by a SBIR grant from The National Institutes of Health (Grant no. R43 DK37214-01).

\section{References}

Agrawal, B.B.L. and Goldstein, I.J. (1966) Specific binding of concanavalin A to cross-linked dextran gels. Biochem. J. $96,23 \mathrm{C}$.

Amante, L., Ancona, A. and Forni, L. (1972) The conjugation of immunoglobulins with tetramethylrhodamine isothiocyanate. A comparison between the amorphous and the crystalline fluorochromes. J. Immunol. Methods. 1, 289.

Bergquist, N.R. and Nilsson, P. (1974) The conjugation of immunoglobulins with tetramethylrhodamine isothiocyanate by utilization of dimethyl sulfoxide (DMSO) as a solvent. J. Immunol. Methods. 5, 189.

Cebra, J.J. and Goldstein, G. (1965) Chromatographic purification of TRITC immunoglobulin conjugates and their use in the cellular localization of rabbit $\gamma$-globulin polypeptide chains. J. Immunol. 95, 230.

Chen, R.F. (1969) Fluorescent protein-dye conjugates. Arch. Biochem. Biophys. 133, 263.

Cherry, R.J., Cogoli, A., Oppliger, M., Schneider, G. and Semenza, G. (1976) A spectroscopic technique for measuring slow rotational diffusion of macromolecules. Biochemistry 15,3653 .

Edelhoch, H. (1967) Spectroscopic determination of tryptophan and tyrosine in proteins. Biochemistry 6, 1948.

Edelman, G.M. and Wang, J.L. (1978) Binding and functional properties of concanavalin $\mathrm{A}$ and its derivatives. J. Biol. Chem. 253, 3016.

Gunther, G.R., Wang, J.L., Yahara, I., Cunningham, B.A. and Edelman, G.M. (1973) Concanavalin A derivatives with altered biological activities. Proc. Natl. Acad. Sci. U.S.A. 70,1012 ,

Haschemeyer, R.H. and Haschemeyer, A.E.V. (1973) Proteins. John Wiley, New York, p. 365.

Ohnishi, S.T. and Barr, J.K. (1978) A simplified method of quantitating proteins using the biuret and phenol reagents. Anal. Biochem. 86, 193.

Ravdin, P. and Axelrod, D. (1977) Fluorescent tetramethylrhodamine derivatives of $\alpha$-bungarotoxin: Preparation, separation, and characterization. Anal. Biochem. 80, 585.

Stryer, L. (1978) Fluorescence energy transfer as a spectroscopic ruler. Ann. Rev. Biochem. 47, 819.

The, T.H. and Feltkamp, T.E.W. (1970) Conjugation of fluorescein isothiocyanate to antibodies. I. Experiments on the conditions of staining. Immunology 18,865 .

Wells, A.F., Miller, G.E. and Nadel, M.K. (1966) Rapid fluorescence and protein assay method for fluorescein-antibody complexes. Appl. Microbiol. 14, 271. 